\title{
Free spin 2 duality invariance cannot be extended to general relativity
}

\author{
S. Deser ${ }^{1, *}$ and D. Seminara ${ }^{2, \dagger}$ \\ ${ }^{1}$ Department of Physics, Brandeis University, Waltham, Massachusetts 02454, USA \\ ${ }^{2}$ Dipartimento di Fisica, Polo Scientifico, Università di Firenze, INFN \\ Sezione di Firenze Via G. Sansone 1, 50019 Sesto Fiorentino, Italy
}

(Received 3 March 2005; published 28 April 2005)

\begin{abstract}
We show by explicit computation that the recently discovered duality invariance of $D=4$ linearized gravity is lost, already at first self-interacting, cubic approximation of general relativity. In contrast, the cubic Yang-Mills extension of Maxwell does admit a simple deformed duality.
\end{abstract}

DOI: 10.1103/PhysRevD.71.081502

PACS numbers: 04.60.Ds, 11.15.- $\mathrm{q}, 11.30 .-\mathrm{j}$

\section{INTRODUCTION}

"Duality" has become a touchstone in relating seemingly different regimes and models in field and string theory. Its humble origins lie in the ancient observation that in (and only in) $D=4$, on-shell configurations with mutually rotated electric-magnetic fields also obey the source-free Maxwell equations. While this observation is essentially correct, it is sometimes misunderstood. Duality can only be properly formulated in terms of the unconstrained dynamical variables, rather than as a formal $\boldsymbol{E} \leftrightarrow$ $\boldsymbol{B}$ rotation [1]. This is already apparent from the facts that $\boldsymbol{B}$ is identically divergenceless, and so can only be related to $\boldsymbol{E}$ upon implementing the latter's Gauss constraint, and from the Maxwell Lagrangian's second order, "hyperbolic," nondual invariant form $L_{M}=\frac{1}{2}\left(\boldsymbol{E}^{2}-\boldsymbol{B}^{2}\right)$.

Surprisingly, duality invariance was recently [2] extended to free massless spin 2, then to free gauge fields of any spin and statistics [3]. Can this Abelian duality invariance be promoted to encompass the two physical self-interacting generalizations, vector [Yang-Mills (YM)] and tensor [general relativity (GR)]? [No consistent non-Abelian higher spin models are known.] The YM conjecture was already considered, and settled in the negative in [1], although we will see that, actually, its first-cubic extension does allow a natural, consistent deformation of duality, at least in Coulomb gauge. Our other, and principal, objective is to settle the question (raised in [2]) of extending duality to GR. We will show (somewhat laboriously) that this cannot be achieved, at least perturbatively: (deformed) duality already fails at its first, interesting, cubic level.

We will begin with a brief summary of what linear duality is (as well as what it is not) for free gauge fields: the 2 degrees of freedom of all $D=4$ free gauge fieldstheir $\pm s$ helicities - can be rotated into one another, by a canonical transformation mixing their two pairs of unconstrained dynamical variables, while keeping the Hamiltonian form-invariant. This is not to be confused with other transformations, such as the "harmonic oscillator" ( $p \rightarrow$

\footnotetext{
*Email address: deser@brandeis.edu

†Email address: seminara@fi.infn.it
}

$q, q \rightarrow-p$ ) rotations within a given mode. We will then revisit the YM system and establish its cubic order duality invariance, before turning to the GR case. An appendix provides details of the latter.

\section{FREE VECTOR FIELD DUALITY}

Free gauge field duality is a canonical transformation, linking coordinates and momenta of different excitations, that leaves the Hamiltonian form-invariant. The simplest case is Maxwell's, whose first-order action is

$$
\begin{aligned}
I_{\max }\left[\boldsymbol{E}^{T}, \boldsymbol{A}^{T}\right]= & \int d^{4} x\left[\left(-\boldsymbol{E}^{T}\right) \cdot \boldsymbol{A}^{T}\right. \\
& \left.-\frac{1}{2}\left(\boldsymbol{E}_{T}^{2}+\left(\boldsymbol{\nabla} \times \boldsymbol{A}^{T}\right)^{2}\right)\right] \\
\equiv & \int d^{4} x\left[\sum_{A=1}^{2} p_{A} \dot{q}^{A}-H\left(p_{A}, q^{A}\right)\right],
\end{aligned}
$$

upon implementing the Gauss constraint, $\boldsymbol{\nabla} \cdot \boldsymbol{E}=0$. Here, transverse vectors are labeled with a " $T$," from the usual transverse-longitudinal decomposition,

$$
\begin{gathered}
\boldsymbol{V}=\boldsymbol{V}^{T}+\boldsymbol{V}^{L}, \quad \boldsymbol{\nabla} \cdot \boldsymbol{V}^{T} \equiv 0 \equiv \nabla \times \boldsymbol{V}^{T}, \\
\int d^{3} x \boldsymbol{V}^{T} \cdot \boldsymbol{W}^{L}=0 .
\end{gathered}
$$

The indicated orthogonality between any pair of $T$ and $L$ vectors implies that only the manifestly gauge-invariant $\boldsymbol{A}^{T}$ component survives in the action (2.1). The duality rotation's infinitesimal form is (we exhibit the-redundant $-T$ index on $\boldsymbol{B}$ for emphasis):

$$
\begin{aligned}
& \delta \boldsymbol{E}^{T}=\boldsymbol{B}^{T} \equiv \boldsymbol{\nabla} \times \boldsymbol{A}^{T}, \\
& \delta \boldsymbol{A}^{T}=\nabla^{-2} \boldsymbol{\nabla} \times \boldsymbol{E}^{T} \Rightarrow \delta \boldsymbol{B}^{T}=-\boldsymbol{E}^{T} .
\end{aligned}
$$

This $\boldsymbol{E}^{T} \leftrightarrow \boldsymbol{B}^{T}$ rotation clearly leaves $\int\left(\boldsymbol{E}_{T}^{2}+\boldsymbol{B}_{T}^{2}\right)$ invariant. The middle equation demonstrates that the desired $\boldsymbol{B}$ rotation is indeed implementable at the level of the canonical coordinates $\boldsymbol{A}^{T}$. That (2.3) is also canonical, i.e., that the symplectic form $S \equiv \int p \dot{q}$ term is invariant, follows from the fact that the curl and Laplacian are Hermitian operators, $\mathcal{O}$, by virtue of which any $\int \chi \mathcal{O} \dot{\chi}=-\int \dot{\chi} \mathcal{O} \chi=$ $-\int \chi \mathcal{O} \dot{\chi}=0$ upon (double) parts integration. The loss of 
manifest Lorentz invariance and of space locality inherent in this procedure is entirely harmless and indeed necessary even to formulate, let alone establish the transformations as canonical ones. [Note that it is only in three space dimensions that the vector $\boldsymbol{E}$ can even be matched with the magnetic tensor $F_{i j}$ (by dualization in the $\varepsilon^{i j k}$ sense).]

The above rotation is quite different from "harmonic oscillator" duality, valid in any dimension

$$
\begin{array}{r}
I[m, k]=\int\left[p \dot{q}-\frac{1}{2}\left(p^{2} / m+k q^{2}\right)\right], \\
\delta p=\alpha q, \quad \delta q=-\beta p, \quad \alpha / \beta=k m,
\end{array}
$$

that relates a single excitation's variables, and reflects the equivalence of different parameter regimes ${ }^{1}$ through the dependence of the solutions on $(\mathrm{k} / \mathrm{m})$. There is a similar duality of Maxwell theory: rewriting (2.1) as

$$
I_{M}=\int\left\{(-\boldsymbol{E}) \cdot \dot{\boldsymbol{A}}-\frac{1}{2}\left[\boldsymbol{E}^{2}+\boldsymbol{A}\left(-\nabla^{2}\right) \boldsymbol{A}\right]\right\},
$$

immediately implies invariance, within each helicity, under

$$
\delta \boldsymbol{E}=\sqrt{-\nabla^{2}} \boldsymbol{A}, \quad \delta \boldsymbol{A}=-\left(-\nabla^{2}\right)^{-1 / 2} \boldsymbol{E} .
$$

\section{CUBIC YANG-MILLS DUALITY}

As an instructive (and transparent) contrast to GR, we study first the nonlinear extension of a Maxwell multiplet by adding the cubic terms in YM, and show that, surprisingly, they permit - a deformed version of - Abelian duality. [This in no way contradicts the demonstration in [1] that full YM precludes duality.] For simplicity, we work with $\mathrm{SU}_{2}$, whose structure constants $\varepsilon^{a b c}$ permit an obvious 3D internal vector notation. The first-order covariant YM action is (setting $g=1$ )

$$
\begin{aligned}
I_{\mathrm{YM}}[\boldsymbol{F}, \boldsymbol{A}] \equiv & -\frac{1}{2} \int\left\{\boldsymbol{F}^{\mu \nu} \cdot\left(\partial_{\mu} \boldsymbol{A}_{\nu}-\partial_{\nu} \boldsymbol{A}_{\mu}+\boldsymbol{A}_{\mu} \times \boldsymbol{A}_{\nu}\right)\right. \\
& \left.-\frac{1}{2} \boldsymbol{F}^{\mu \nu} \cdot \boldsymbol{F}_{\mu \nu}\right\} .
\end{aligned}
$$

The $(3+1)$ versions of (3.1) and of the Gauss constraint become

$$
\begin{aligned}
& I_{\mathrm{YM}}[\boldsymbol{E}, \boldsymbol{A}]=\frac{1}{2} \int\left[\left(-\boldsymbol{E}^{T}\right) \cdot \dot{\boldsymbol{A}}-\frac{1}{2}\left(\boldsymbol{E}^{2}+\boldsymbol{B}^{2}\right)\right], \\
& \boldsymbol{B} \equiv \boldsymbol{\nabla} \times \boldsymbol{A}+\boldsymbol{A} \times \boldsymbol{A}, \quad \boldsymbol{D} \cdot \boldsymbol{E} \equiv(\boldsymbol{\nabla}+\boldsymbol{A} \times) \cdot \boldsymbol{E}=0 .
\end{aligned}
$$

Adopting Coulomb gauge, $\boldsymbol{A}^{L}=0$ simplifies the process:

$$
I_{\mathrm{YM}}=\frac{1}{2} \int\left\{\left(-\boldsymbol{E}^{T}\right) \cdot \dot{\boldsymbol{A}}_{T}-\frac{1}{2}\left[\boldsymbol{E}_{T}^{2}+\boldsymbol{E}_{L}^{2}+\boldsymbol{B}^{2}\right]\right\},
$$

\footnotetext{
${ }^{1}$ Or relating different models, here $I[m, k] \leftrightarrow I\left[k^{-1}, m^{-1}\right]$, with different parameters [4].
}

$$
\left(\boldsymbol{\nabla}+\boldsymbol{A}_{T} \times\right) \cdot \boldsymbol{E}_{L}=-\boldsymbol{A}_{T} \times \boldsymbol{E}_{T} .
$$

The constraint ${ }^{2}$ fixes $\boldsymbol{E}_{L}$ in terms of the dynamical pairs: $\boldsymbol{E}_{L}=0+\mathcal{O}\left(\boldsymbol{A}^{T} \boldsymbol{E}^{T}\right)$; since $\boldsymbol{E}_{L}^{2}$ is quartic, we may drop it from (3.3) to cubic order; omitting " $T$," this leaves

$$
\begin{aligned}
I_{\mathrm{YM}}[\boldsymbol{E}, \boldsymbol{A}]= & I_{M}[\boldsymbol{E}, \boldsymbol{A}]+I_{\mathrm{YM}}^{c}[\boldsymbol{E}, \boldsymbol{A}] \\
\cong & \frac{1}{2} \int\left\{(-\boldsymbol{E}) \cdot \dot{\boldsymbol{A}}-\frac{1}{2}\left(\boldsymbol{E}^{2}+(\boldsymbol{\nabla} \times \boldsymbol{A})^{2}\right\}\right. \\
& +\int \boldsymbol{\nabla} \times \boldsymbol{A} \cdot \boldsymbol{A} \times \boldsymbol{A} .
\end{aligned}
$$

Only the final term in $H$ differentiates the action from that of a triplet of photons. The original linear duality transformation (2.3) clearly alters (only) this term,

$$
\delta_{L} \int(\nabla \times \boldsymbol{A} \cdot \boldsymbol{A} \times \boldsymbol{A})=3 \int \boldsymbol{E} \cdot \boldsymbol{A} \times \boldsymbol{A} \neq 0 .
$$

To cancel this cubic term, we must deform the Abelian transformation by adding a quadratic $\delta_{Q} \boldsymbol{E}$, that will generate a cubic variation from the $\int \boldsymbol{E}^{2}$ in (3.5); the obvious choice is

$$
\delta_{Q} \boldsymbol{E}=(\boldsymbol{A} \times \boldsymbol{A})^{T}
$$

[We have projected the " $T$ " part of $(\boldsymbol{A} \times \boldsymbol{A})$ since $\boldsymbol{E}$ is transverse; this is just a formality here, and throughout, since orthogonality would automatically perform the projection in $\int \boldsymbol{E}^{T} \cdot \delta_{Q} \boldsymbol{E}$.] Having succeeded in keeping $H_{\mathrm{YM}}$ invariant, we must still check that the symplectic variation, generated by (3.7), namely

$$
\delta_{Q} \int \boldsymbol{E} \cdot \dot{\boldsymbol{A}}=\int \boldsymbol{A} \times \boldsymbol{A} \cdot \dot{\boldsymbol{A}},
$$

vanishes. Indeed, time integration by parts shows it to equal minus twice itself.

To summarize, we have succeeded in keeping duality invariance of YM to lowest nonlinear order, in $\boldsymbol{A}_{L}=0$ gauge at least. This was accomplished by setting $\delta \boldsymbol{E}=$ $\boldsymbol{B} \equiv \boldsymbol{\nabla} \times \boldsymbol{A}+\boldsymbol{A} \times \boldsymbol{A}$, certainly the most obvious guess; the corresponding part of the generator is just the $\mathrm{YM}$ Chern-Simons form:

$$
\begin{aligned}
& G_{E}=\int d^{3} x\left[\boldsymbol{A} \cdot \boldsymbol{\nabla} \times \boldsymbol{A}+\frac{2}{3} \boldsymbol{A} \cdot \boldsymbol{A} \times \boldsymbol{A}\right], \\
& \delta \boldsymbol{E}=\left[G_{E}, \boldsymbol{E}\right]=\boldsymbol{\nabla} \times \boldsymbol{A}+\boldsymbol{A} \times \boldsymbol{A} \equiv \boldsymbol{B}_{\mathrm{YM}} .
\end{aligned}
$$

We have not attempted to extend this process to quartic order, where terms $\sim(\boldsymbol{A} \times \boldsymbol{A})^{2}$ from $\boldsymbol{B}^{2}$ and $\sim\left(\boldsymbol{A} \cdot \boldsymbol{E}_{T}\right)^{2}$ from $\boldsymbol{E}_{L}^{2}$ appear, nor do we know whether the cubic order success has some deeper physical origin.

\section{GR DUALITY FAILS}

Having illustrated how duality deformations can succeed at cubic level for YM vectors, we now turn to theconsiderably more complicated-tensor case. We will

\footnotetext{
${ }^{2}$ Formally, inversion of (3.4) for $\boldsymbol{E}_{L}\left[\boldsymbol{A}_{T}, \boldsymbol{E}_{T}\right]$ would solve classical YM entirely.
} 
begin with a description of Pauli-Fierz free spin 2 theory, transcribed into a notation manifesting its duality invariance. We will then derive the cubic correction to GR, and subject it to the Abelian transformations. Finally, we will show that its lack of invariance under the latter cannot be compensated by adding further, quadratic deformations of the dynamical variables: there is no (perturbative) duality invariance in GR.

Let us first express the familiar free spin 2 gauge system in "dual-ready" form, using the first-order formulation of full GR [5], thereby also obtaining the cubic correction in a unified way. The Einstein action is

$$
\begin{aligned}
I_{E}[\pi, g] & =\int d^{4} x\left[\pi^{i j} \dot{g}_{i j}-N_{\mu} R^{\mu}\right], \\
N_{0} & \equiv\left(-g^{00}\right)^{-1 / 2}, \quad N_{i} \equiv g_{0 i}, \\
R^{0} & \equiv-\sqrt{{ }^{3} g^{3}} R+\left(\pi_{i j} \pi^{i j}-\frac{1}{2} \pi_{i}^{i} \pi_{j}^{j}\right)\left({ }^{3} g\right)^{-1 / 2}, \\
R^{i} & \equiv-2 D_{j} \pi^{i j} .
\end{aligned}
$$

All explicit roman indices refer to the intrinsic 3-space, of which ${ }^{3} R$ is the scalar curvature and $D_{j}$ the covariant 3 derivative; $\pi^{i j}$, essentially the second fundamental form (density), is an independent variable on a par with $g_{i j}$. Our convention is $R_{i j} \sim+\partial_{k} \Gamma_{i j}^{k}$. The expansion about flat space is $^{3}$

$$
h_{i j} \equiv g_{i j}-\delta_{i j}, \quad N_{0}=1+n ;
$$

instead, $N_{i}$ and $\pi^{i j}$ vanish in flat space and are of first order. Using Abelian gauge invariance and the four constraints, $R^{\mu}=0$ simplifies (4.1) dramatically. We recall the familiar transverse-traceless orthogonal decomposition of a flat space (where index position is immaterial) spatial tensor $T_{i j}=T_{j i}$,

$$
\begin{aligned}
T_{i j}= & T_{i j}^{T T}+\frac{1}{2}\left(\delta_{i j}-\nabla^{-2} \partial_{i j}^{2}\right) T^{T} \\
& +\left(\partial_{i} T_{j}+\partial_{j} T_{i}\right), \\
\partial_{i} T_{i j}^{T T}= & 0=T_{i i}^{T T}, \\
\partial_{j}\left(\delta_{i j}-\nabla^{-2} \partial_{i j}^{2}\right) \equiv & 0 \equiv\left(\delta_{i j}-\nabla^{2} \partial_{i j}^{2}\right) \partial_{j} .
\end{aligned}
$$

Of the six components of $T_{i j}$, two are " $T T$," one is " $T$," and three represent the vector $T_{i}$. Orthogonality between the various components under integration is manifest; one example is $\int d^{3} x T_{i j}^{T T} \partial_{i} W_{j}=0$. Once decomposed in this fashion, the $12\left(\pi^{i j}, h_{i j}\right)$ components are easily classified: at linearized level, the theory's Abelian invariance under $\delta h_{\mu \nu}=\left(\partial_{\mu} \xi_{\nu}+\partial_{\nu} \xi_{\mu}\right)$ simply means that the four gauge variables $\left(\pi^{T}, h_{i}\right)$ do not appear in the quadratic action. Likewise, the four linearized constraints,

\footnotetext{
${ }^{3}$ We spell out the fact that $g_{i j}$ is exactly $\delta_{i j}+h_{i j}$ to all orders, so that neither $h_{i j}$ nor $\pi^{i j}$ are subject to further expansion, unlike say $g^{i j} \sim \delta_{i j}-h^{i j}+\mathcal{O}\left(h^{2}\right)$ or $\sqrt{3} g \sim 1+\frac{1}{2} h_{i i}+\mathcal{O}\left(h^{2}\right)$.
}

$$
\begin{aligned}
-R^{0} \rightarrow{ }^{3} R_{L}(h) & \equiv\left(\partial_{i j}^{2} h_{i j}-\nabla^{2} h_{i i}\right)=-\nabla^{2} h^{T}=0, \\
R^{i} & \rightarrow-2 \partial_{j} \pi^{i j}=0
\end{aligned}
$$

remove the respective components $\left(h^{T}, \pi^{i}\right)$, as is also obvious for $\pi^{i}$ upon using (4.3). The four remaining $\left(\pi^{i j}, h_{i j}\right)$ then simply reduce to their two pairs of " $T T$ " degrees of freedom. We designate them by ${ }^{4}\left(p^{i j}, h_{i j}\right)$ henceforth dropping the "TT" notation. The symplectic term is just that of the two excitations,

$$
S=\int d^{4} x \pi^{i j} \dot{g}_{i j} \rightarrow \int d^{4} x p^{i j} \dot{q}_{i j},
$$

while the Hamiltonian is simply the quadratic part of $R^{0}$, subject to the linear constraints. A short calculation (essentially integrations), finally reduces (4.1) to the usual harmonic oscillator form,

$$
I_{E}^{Q}[p, q]=\int d^{4} x\left\{\sum_{A=1}^{2} p_{A} \dot{q}^{A}-\frac{1}{2}\left[p^{2}+(\nabla q)^{2}\right]\right\},
$$

with traces on implicit indices understood. For our purposes, the notion of duality in this symmetric tensor world emerges from the existence of a generalized curl [3], distributed on the tensor's indices:

$$
(\mathcal{O} T)_{i j} \equiv \frac{1}{2}\left(\varepsilon^{i \ell m} \partial_{\ell} T_{m j}+\varepsilon^{j \ell m} \partial_{\ell} T_{i m}\right)=(\mathcal{O} T)_{j i} .
$$

Its nonlocal extension

$$
(\tilde{\mathcal{O} T})_{i j} \equiv\left(\nabla^{-2} \mathcal{O} T\right)_{i j}
$$

will also streamline notation; both $\mathcal{O}$ and $\tilde{\mathcal{O}}$ operations are Hermitian. Thus, acting on $q_{i j}$ the curl defines a "magnetic" field,

$$
B_{i j} \equiv(\mathcal{O} q)_{i j}=B_{j i}
$$

in terms of which (4.6) becomes

$$
I_{E}^{Q}[p, q]=\int d^{4} x\left\{p \dot{q}-\frac{1}{2}\left(p^{2}+B^{2}\right)\right\} .
$$

This form makes it irresistible to perform the rotation ${ }^{5}$

$$
\delta p_{i j}=B_{i j}, \quad \delta q_{i j}=(\tilde{\mathcal{O}} p)_{i j} \Rightarrow \delta B_{i j}=-p_{i j}
$$

the final step uses $\mathcal{O O}=-\nabla^{2}$, just as $\nabla \times \nabla \times=-\nabla^{2}$ on transverse vectors. This transformation obviously maintains the Hamiltonian of (4.6), while the $q$ sector of (4.10) shows that $\delta B$ is indeed implementable as a transformation of the underlying coordinate $q$. Invariance of the symplectic term is guaranteed (as for spin 1) by Hermiticity of the $\tilde{\mathcal{O}}$ and $\mathcal{O}$ operators.

\footnotetext{
${ }^{4}$ More precisely, the original linear Hamiltonian is $\int\left[\pi_{i j}^{2}+\right.$ $\left.\frac{1}{4}\left(\nabla h_{i j}\right)^{2}\right]$, requiring the rescaling $\sqrt{2} \pi^{i j}=p^{i j}, 1 / \sqrt{2} h_{i j}=q_{i j}$ to bring it into standard free-field form (4.6).

${ }^{5}$ Higher free spin fields [3] follow the same pattern, in terms of suitably generalized curl operations to accommodate any number of spatial indices.
} 
Establishing duality transformation and invariance of free spin 2 under them was the easy part; now we must return to the full action (4.1) to obtain its cubic, $I_{E}^{c}[p, q]$, correction, subject it to the linear rotations (4.10), and try to cancel away their (nonvanishing) effect by quadratically deforming them.

The cubic correction to $H$ is formally easy to find. It is simply the cubic part, $R_{c}^{0}$, subject to the lower level constraints. The procedure is greatly simplified, as it was for YM, by choosing "Coulomb" gauge; here $h_{i}=0=\pi^{T}$. [Recall that only the linearized action is invariant under the linear part of the non-Abelian $\delta g_{\mu \nu}=D_{\mu} \xi_{\nu}+D_{\nu} \xi_{\mu}$.] The upshot of this process is the cubic Hamiltonian

$$
\begin{aligned}
H_{c}(p, q)= & R_{c}^{0}(p, q) \\
= & \int d^{3} x\left[\left(-{ }^{3} R \sqrt{g}\right)_{c}+2 p^{i j} p^{\ell m}\right. \\
& \left.\times\left(q_{i \ell} \delta_{j m}-\frac{1}{2} q_{i j} \delta_{\ell m}\right)\right] ;
\end{aligned}
$$

the last term in (4.11) vanishes due to $p^{\ell m} \delta_{\ell m}=0$. Furthermore, the symplectic term has the great virtue of remaining quadratic in our gauge, while the lower constraints also imply that the same $(p, q)$ are dynamical.

Whereas the cubic order curvature scalar density is a bit elaborate, we are in fact only interested in its (linear) variation;

$$
\delta_{L} \int\left({ }^{3} R \sqrt{g}\right)_{c}=-\int\left(\sqrt{g} G^{i j}\right)_{Q} \delta_{L} q_{i j},
$$

by the Palatini identity. The variation of the cubic kinetic terms is just $\delta_{L} \int 2 \operatorname{trppq}$, so we have

$$
\begin{aligned}
\delta_{L} H_{c}(p, q)= & \int d^{3} r G_{i j}^{Q} \delta_{L} p^{i j}+2 \int(p p)_{i j} \delta_{L} q_{i j} \\
& +2 \int\left\{\delta_{L} p^{i j}(p q)_{i j}+\left(p \delta_{L} p\right)_{i j} q_{i j}\right\} .
\end{aligned}
$$

Schematically, the variation (4.13) is of the form $\delta_{L} I \sim$ $q^{2} p+p^{3}$. There is an a priori daunting array of possible compensating quadratic deformations of each $(p, q)$ : $\delta_{Q}(p, q) \sim\left(p^{2}+p q+q^{2}\right)$, with various possible derivative and index structures in each. Further, all are subject to keeping the relative dimensions of $(p \sim \dot{q}, q)$ as well as enforcing $\delta \int p \dot{q}=0$. Owing to the dimensional asymmetry between $\delta_{Q} p$ and $\delta_{Q} q$, the latter will involve the nonlocal operator $\tilde{\mathcal{O}}$, as compared to $\delta_{Q} p \sim \mathcal{O}$. This will lead to the presence of both local and nonlocal variations that must separately cancel.

[Parenthetically, we lay to rest the otherwise attractive idea that, since duality invariance requires the other Poincaré generators of the cubic model to be invariant too, one could test the momentum generator instead. Unfortunately, Dirac's dictum that the Hamiltonian form of dynamics always has a simple momentum holds here as well. The transformation generator $G=P_{i} \delta x^{i}$, with $P_{i}=$
$-2 \int \partial_{j} \pi_{i}^{j}$ and the constraint $D_{j} \pi^{i j}=0$, says that to all orders

$$
\partial_{j} \pi_{i}^{j}=\Gamma_{j i}^{k} \pi_{k}^{j}=[j i, k] \pi^{j k}=\frac{1}{2} p \nabla_{i} q .
$$

Hence $\boldsymbol{P}=\int p \boldsymbol{\nabla} q$ to all orders [5] and its invariance provides no independent test beyond that of $\int p \frac{\partial}{\partial t} q$. The same holds for the rotation generator, of course.]

In the following, we will just outline the flow of possible deformations and their consequences. The more unpleasant details are relegated to the appendix. Returning to (4.13), we have

$$
\begin{aligned}
\delta_{L} H_{c}(p, q)= & \int d^{3} r\left[G_{Q}^{i j}(\tilde{\mathcal{O}} p)_{i j}+2 \operatorname{trp} p \tilde{\mathcal{O}} p\right. \\
& +2 \operatorname{Tr}\{q p \mathcal{O} q+q \mathcal{O} q p\}] \sim p^{3}+q^{2} p .
\end{aligned}
$$

Obviously, the simplest term to cancel is the pure $p^{3}$, which can (only) be accomplished by $\delta_{Q}^{(1)} p \sim p^{2}$. More precisely, acting on the quadratic Hamiltonian, this deformation leads to ${ }^{6}$

$$
\delta_{Q}^{(1)} p_{i j} \sim(p \tilde{\mathcal{O}} p)_{i j} \Rightarrow \delta_{Q}^{(1)} \int p^{2} \sim \operatorname{Tr}(p p \tilde{\mathcal{O}} p),
$$

exactly of the form of the term to be canceled. Now, however, we have to cancel the unwanted effect of (4.16) on $\int p \dot{q}$,

$$
\delta^{(1)} \int p \dot{q} \sim \operatorname{Tr} \int(p \tilde{\mathcal{O}} p)_{i j} \dot{q}_{i j} .
$$

The unique possible cure for this is

$$
\delta_{Q}^{(1)} q \sim(q \tilde{\mathcal{O}} p+p \tilde{\mathcal{O}} q)_{i j} ;
$$

and indeed it works, canceling (4.17). While we have now gotten rid of the $p^{3}$ part of (4.15), we must not forget one further effect, that of (4.18) on $H_{Q}$,

$$
\delta_{Q}^{(1)} \frac{1}{2} \int q_{i j}\left(-\nabla^{2}\right) q_{i j} \sim \operatorname{Tr} \int q\left(-\nabla^{2}\right)[q \tilde{\mathcal{O}} p+p \tilde{\mathcal{O}} q] \sim q^{2} p,
$$

a generically nonlocal term. The possible remedies to the overall $\int q^{2} p$ residue of (4.15) plus (4.19) consist of $\delta_{Q}^{(2)} p \sim q^{2}$ and $\delta_{Q}^{(2)} q \sim q p$. The latter choice is clearly not desirable, since we have just exploited it in (4.18), in an essentially unique way. We are therefore stuck with the last hope,

$$
\delta_{Q}^{(2)} p \sim q \mathcal{O} q, \quad \delta_{Q}^{(2)} \frac{1}{2} \int p^{2}=\operatorname{Tr} \int p(q \mathcal{O} q) .
$$

\footnotetext{
${ }^{6}$ Here, and in all quadratic transformations, a projection to " $T T$ " space is understood, to keep the character of $(p, q)$. However, this is really immaterial, since they always safely multiply a linear $T T$ variable.
} 
Now we exploit the local/nonlocal division of the variations. In (4.15) there are both kinds; the former type is easy, since (by dimensions) it has no explicit derivative beyond the $\operatorname{curl} \mathcal{O}$, that is,

$$
\delta_{\text {local }} H_{c} \sim 2 \int\{p(\mathcal{O} q) q+q \mathcal{O} q p\}
$$

This is clearly canceled by (and only by)

$$
\delta_{Q}^{(2)} p \sim 4(q \mathcal{O} q), \quad \delta \frac{1}{2} \int p^{2}=4 \operatorname{Tr} \int p(\mathcal{O} q) q .
$$

The symplectic contribution of (4.22) "miraculously" vanishes; its (local) form is

$$
\delta_{\text {local }}^{(2)} \int p \dot{q} \sim \operatorname{Tr} \int(q q)_{i j}(\mathcal{O} \dot{q})_{i j}
$$

The positions of the dot and the curl are immaterial. What matters is that $\mathcal{O}$ is Hermitian, while $\partial / \partial t$ changes sign on integration by parts, which suffices to show this term is proportional to minus itself and vanishes. The (decisive) nonlocal terms require the appendix.

\section{SUMMARY}

We have considered whether any deformations of the duality transformations of linearized vector and tensor gauge theories might rescue (an extended version of) duality in their nonlinear regimes. ${ }^{7}$

For YM, it was surprisingly easy, at least in Coulomb gauge, to do so at leading, cubic, "post-Maxwell" order, simply by letting the electric field variable rotate into the full YM magnetic field, while keeping the vector potential's rotation unaltered. We have not explicitly analyzed quartic order and beyond in the theory's infinite series expansion in $(p, q)$, since it is known that duality fails for full YM.

Extension of free spin 2 duality to cubic order proved considerably more complicated to decide, but we were able to show that no deformation compensates for the loss of Abelian duality invariance, even in Coulomb gauge. We conclude that, perhaps disappointingly, rotation among helicities ceases to be an invariance beyond the free spin 2 level.

\section{ACKNOWLEDGMENTS}

This work was supported in part by NSF Grant No. PHY04-01667.

\footnotetext{
${ }^{7}$ The vector and tensor models are of course very different, not only in index complication, but in the fact that cubic and higher YM terms are of lower derivative order, whereas in GR, all orders have two derivatives.
}

\section{APPENDIX}

In Sec. IV, we sketched problems of extending linear spin 2 duality to the cubic term of GR. This appendix provides details of the obstacles and shows why they cannot be overcome. We first collect the key quantities, starting with the gravity Hamiltonian at quadratic,

$$
H_{Q}=\frac{1}{2} \int d^{3} x\left[(\nabla q)^{2}+p^{2}\right]
$$

and cubic,

$$
H_{c}(p, q)=\int d^{3} x\left[-{ }^{3} R_{c}(q)+2 p^{i}{ }_{m} p^{\ell m} q_{i \ell}\right],
$$

levels, with all relevant constraints satisfied: only " $T T$ " variables are involved. [The corrections coming from solving the nonlinear constraints affect only quartic terms.] The Abelian duality transformation (4.10), which preserves $H_{Q}$, is

$$
\delta^{(1)} q_{a b}=(\tilde{\mathcal{O}} p)_{a b} \equiv P_{a b} \quad \delta^{(1)} p^{a b}=(\mathcal{O} q)_{a b} \equiv Q_{a b} .
$$

We now consider the effect of this rotation on $H_{c}$, starting with the variation of the simple cubic vertex $p p q$ of (A2)

$$
\begin{aligned}
-2 \delta \int p p q & =-2 \int \delta^{(1)} q_{a b} p^{a c} p_{c}^{b}+2 q_{a b} \delta^{(1)} p^{a c} p_{c}^{b} \\
& \equiv A+B .
\end{aligned}
$$

The $A$ term's contribution in the above variation is proportional to $p^{3}$. Specifically we have

$$
-2\left(\delta^{(1)} q_{a b}\right) \pi^{a c} \pi_{c}^{b}=P_{a b}(p p)_{a b} .
$$

The unique and obvious way to cancel the above variation introduces a contribution in $\delta p$ quadratic in momentum,

$$
\delta^{(2)} p_{b c}=-P_{a b} p_{b c} .
$$

The ensuing variation of the $p^{2}$ term in $H_{Q}$ then exactly cancels (A5). This deformation of $\delta p$ obviously affects the symplectic form $S$, which in turn must be compensated. Indeed,

$$
\delta S=\int d^{4} x \delta^{(2)} p^{a b} \dot{q}_{a b}=-\int d^{4} x P_{a b} p_{b c} \dot{q}_{a c} .
$$

Canceling this variation requires, and completely fixes, a quadratic term $\sim p q$ in $\delta q_{a b}$. [Any alteration would automatically produce a change in the $p^{2}$ term $\delta^{(2)} p^{a b}$, which, however, has just been determined.] To evaluate the required $q p$ term in $\delta^{(2)} q_{a b}$, we first integrate (A7) by parts with respect to time,

$$
\delta S=\int d^{4} x \operatorname{Tr}[q P \dot{p}+(p q) \dot{P}] .
$$

Thus, the desired variation must be

$$
\delta_{q p}^{(2)} q_{a c}=\left[q_{c b} P_{b a}+(a b)\right] .
$$


Next we consider the $B$ term in (A4), namely

$$
-4 \int d^{3} x q_{a b}\left(\delta^{(1)} p^{a c}\right) p_{c}^{b}=-4 \int d^{3} x \operatorname{Tr}(q Q p) .
$$

Variations of the same $p q^{2}$ form will also contribute from ${ }^{3} R_{c}(q)$ in (A2),

$$
\begin{aligned}
-\delta \int d^{3} x^{(3)} R & =-\int d^{3} x^{(2)} G^{a b}(q) \delta^{(1)} q_{a b} \\
& =2 \int d^{3} x R_{Q}^{a b} P_{a b},
\end{aligned}
$$

as well as from the $q^{2}$ term in $H_{Q}$,

$$
-\frac{1}{2} \int d^{3} x q_{a b} \nabla^{2} \delta_{q p}^{(2)} q^{a b}=-\frac{1}{2} \int d^{3} x \operatorname{Tr}\left[q \nabla^{2} q P+q Q p\right] .
$$

The variation in (A11) can be rearranged by explicitly displaying the Ricci tensor ${ }^{8}$ to $\mathcal{O}\left(q^{2}\right)$ :

$$
\begin{aligned}
{ }^{(2)} R^{a b}= & \left(\frac{1}{2} q_{a c} \nabla^{2} q_{b}^{c}+\frac{1}{2} q_{b c} \nabla^{2} q_{a}^{c}\right) \\
& -\frac{1}{2} \partial_{r}\left(q^{r n}\left(\partial_{a} q_{n b}+\partial_{b} q_{n a}-\partial_{n} q_{a b}\right)\right) \\
& +\frac{1}{4} \partial_{a} \partial_{b}\left(q^{r n} q_{r n}\right)-\frac{1}{4} \partial_{a} q_{m r} \partial_{b} q^{m r} \\
& -\frac{1}{4}\left(\partial^{r} q_{b}^{m}-\partial^{m} q_{b}^{r}\right)\left(\partial_{m} q_{a r}-\partial_{r} q_{a m}\right) .
\end{aligned}
$$

The $\delta_{a} \delta_{b}$ term can be dropped because it is longitudinal and vanishes when integrating with a $T T$ object. The variation (A11) can now be rewritten as follows:

$$
\begin{aligned}
2 \int d^{3} x R_{Q}^{a b}(q) P_{a b}= & \int d^{3} x\left[q_{a c} \nabla^{2} q_{b}^{c}+q_{b c} \nabla^{2} q_{a}^{c}\right. \\
& -\partial_{l}\left(q^{l n}\left(\partial_{a} q_{n b}+\partial_{b} q_{n a}-\partial_{n} q_{a b}\right)\right) \\
& -\frac{1}{2} \partial_{a} q_{m l} \partial_{b} q^{m l}-\left(\partial_{m} q_{a l} \partial^{l} q_{b m}\right. \\
& \left.\left.-\partial_{m} q_{a l} \partial^{m} q_{b}^{l}\right)\right] P_{a b} .
\end{aligned}
$$

The combination of the variation (A12) and (A14) simplifies a bit to

$$
\begin{aligned}
\int d^{3} x[\{ & \left(-\partial_{l}\left(q^{l n}\left(\partial_{a} q_{n b}+\partial_{b} q_{n a}-\partial_{n} q_{a b}\right)\right)\right. \\
& \left.-\frac{1}{2} \partial_{a} q_{m l} \partial_{b} q^{m l}-\partial_{m} q_{a l} \partial^{l} q_{b m}\right\} P_{a b} \\
& \left.+\frac{1}{2}\left(q_{a c} q_{b}^{c}\right) P_{a b}-\frac{1}{2} q_{b}^{m} Q_{a b} p_{a m}\right] .
\end{aligned}
$$

Finally, inclusion of (A10) yields the complete cubic variation,

\footnotetext{
${ }^{8}$ We are allowed to drop $\left(g^{a b} R\right)^{Q}=\delta^{a b} R^{Q}-h_{a b} R_{L}$; the first of these vanishes when contracted with a $T T$ tensor, the second because $R_{L}=0$ is the linear constraint.
}

$$
\begin{aligned}
\delta_{\mathrm{tot}} H= & \int d^{3} x\left[\left\{\left(-\partial_{l}\left(q^{l n}\left(\partial_{a} q_{n b}+\partial_{b} q_{n a}-\partial_{n} q_{a b}\right)\right)\right.\right.\right. \\
& \left.-\frac{1}{2} \partial_{a} q_{m l} \partial_{b} q^{m l}-\partial_{m} q_{a l} \partial^{l} q_{b m}\right\} P_{a b} \\
& \left.+\frac{1}{2} \operatorname{tr}(q q P)+\frac{1}{2} q_{b}^{m} Q_{a b} p_{a m}\right] .
\end{aligned}
$$

In order to cancel this variation, as already explained in text, we may only modify $\delta^{(2)} p$ by adding to it terms proportional to $q^{2}$, while preserving the symplectic form without help from other sectors. It produces, in the variation of the symplectic form, a contribution of the type $q^{2} \dot{q}$ that cannot originate from anywhere else.

In this remaining variation (A16), we see two "noninteracting" sectors: the first is nonlocal $\left(\nabla^{-2}\right)$ and the second local; this splitting is unambiguous. Inspecting the position of the indices of the derivatives in the nonlocal part, one sees that they cannot produce further local contribution by integration by parts. Hence the two contributions must be canceled by separate terms in the variation $\delta^{(2)} p_{a b}$. Let us dispose first of the local sector. Here, we modify $\delta^{(2)} p_{a b}$ as follows:

$$
\delta_{q^{2}}^{(2)} p^{a b}=\mathcal{O}(q q)_{a b}-q_{a c} Q_{c b}+(b \leftrightarrow a) .
$$

The resulting variation of the symplectic form vanishes.

$$
\begin{aligned}
\delta_{q^{2}} S= & \int d^{4} x\left(\frac{1}{2} \dot{q}_{a b} \epsilon^{m r a} \partial_{r}\left(q_{m c} q^{c b}\right)\right. \\
& \left.-\frac{1}{2} \epsilon^{m r a} \partial_{r}\left(q_{m c} q^{c b}\right) \dot{q}_{a b}\right)=0 .
\end{aligned}
$$

As explained in the text, any term $\sim \int(\mathcal{O} \dot{q}) q q$ effectively changes sign upon simultaneous space and time integrations by part. This brings us down to the nonlocal part, the final (and as we now see, incurable) obstacles:

$$
\begin{aligned}
\delta_{\mathrm{NL}} H_{c}= & -\int d^{3} x\left[\partial_{l}\left(q^{l n}\left(\partial_{a} q_{n b}+\partial_{b} q_{n a}-\partial_{n} q_{a b}\right)\right)\right. \\
& \left.+\frac{1}{2} \partial_{a} q_{m l} \partial_{b} q^{m l}+\left(\partial_{m} q_{a l} \partial^{l} q_{b m}\right)\right] P_{a b} .
\end{aligned}
$$

This proliferation of terms represents the additional index possibilities once the extra $\nabla^{-2}$ brings in two extra derivatives in the numerator.

Write (A19) schematically as

$$
\delta_{\mathrm{NL}} H_{c}=\int d^{3} x[q q] P \equiv \int d^{3} x p \tilde{\mathcal{O}}[p p] .
$$

As in the local term's analysis, (A20) can be canceled by $\delta_{\mathrm{NL}}^{(2)} p_{a b}=\{\tilde{\mathcal{O}}[q q]\}_{a b}$ acting on the $p^{2}$ part of $H_{Q}$, and the last hurdle is the ensuing symplectic variation, engendered by $\delta_{\mathrm{NL}}^{(2)} p$ :

$$
\delta_{\mathrm{NL}} S=\int q q \tilde{\mathcal{O}} \dot{q},
$$

which must again vanish by itself, there being no further 
variations left to help it. From (A19), then,

$$
\begin{aligned}
\delta_{\mathrm{NL}} S= & \int d^{4} x\left\{\partial_{\ell m}\left(q_{a \ell} q_{b m}-q_{a b} q_{\ell m}\right)\right. \\
& \left.+\frac{1}{2} q_{m \ell, a} q_{m \ell, b}+2 \partial_{\ell}\left(q_{\ell m} q_{m a, b}\right)\right\} \tilde{\mathcal{O}} \dot{q}_{a b} .
\end{aligned}
$$

The terms in curly brackets are essentially all the possible forms involving $q q$ and $\partial \partial$ as a 2-index tensor: two, one, and no "dummy" derivatives. Unlike in the local term's $q q \dot{q}$ there are no cancellations here and indeed there are concrete counterexamples of $T T q$ tensors with $\delta_{\mathrm{NL}} S \neq 0$.
[1] S. Deser and C. Teitelboim, Phys. Rev. D 13, 1592 (1976); S. Deser, J. Phys. A 15, 1053 (1982).

[2] M. Henneaux and C. Teitelboim, Phys. Rev. D 71, 024018 (2005).

[3] S. Deser and D. Seminara, Phys. Lett. B 607, 317 (2005).
[4] B. Zwiebach, A First Course in String Theory (Cambridge University Press, Cambridge, England, 2004), p. 387.

[5] R. Arnowitt, S. Deser, and C. Misner, in Gravitation: An Introduction to Current Research, edited by L. Witten (Wiley, New York, 1962). 